NASA Technical Memorandum

107740

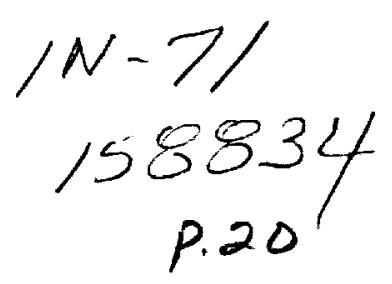

ON THE EFFECT OF ACOUSTIC COUPLING ON RANDOM AND HARMONIC PLATE VIBRATIONS

A. FRENDI

ANALYTICAL SERVICES \& MATERIALS, INC. HAMPTON, VIRGINIA

J. H. ROBINSON

NASA LANGLEY RESEARCH CENTER

HAMPTON, VIRGINIA

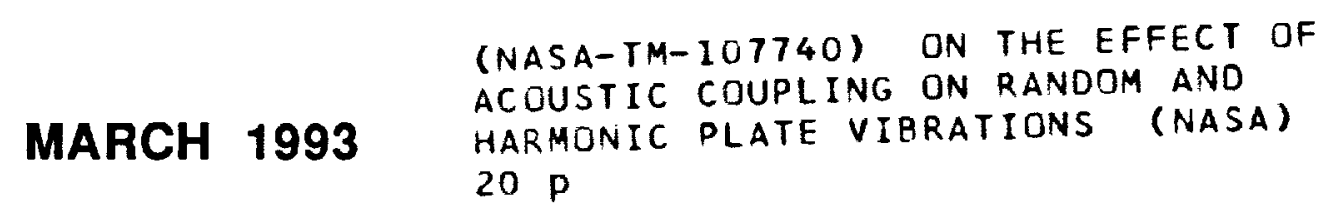

N93-25189

ACOUSTIC COUPLING ON RANDOM AND

HARMONIC PLATE VIBRATIONS

G3/71 0158834 
,

- 


\title{
ON THE EFFECT OF ACOUSTIC COUPLING ON RANDOM AND HARMONIC PLATE VIBRATIONS
}

\author{
Abdelkader Frendi \\ Analytical Services and Material Inc. \\ Hampton, VA 23666 \\ Jay Robinson \\ NASA Langley Research Center \\ Hampton, VA 23681-0001
}

\begin{abstract}
The effect of acoustic coupling on random and harmonic plate vibrations is studied using two numerical models. In the coupled model, the plate response is obtained by integration of the nonlinear plate equation coupled with the nonlinear Euler equations for the surrounding acoustic fluid. In the uncoupled model, the nonlinear plate equation with an equivalent linear viscous damping term is integrated to obtain the response of the plate subject to the same excitation field. For a low-level, narrow-band excitation, the two models predict the same plate response spectra. As the excitation level is increased, the response power spectrum predicted by the uncoupled model becomes broader and more shifted towards the high frequencies than that obtained by the coupled model. In addition, the difference in response between the coupled and uncoupled models at high frequencies becomes larger. When a high intensity harmonic excitation is used, causing a nonlinear plate response, both models predict the same frequency content of the response. However, the level of the harmonics and subharmonics are higher for the uncoupled model. Comparisons to earlier experimental and numerical results show that acoustic coupling has a significant effect on the plate response at high excitation levels. Its absence in previous models may explain the discrepancy between predicted and measured responses.
\end{abstract}




\section{Introduction}

It is well known in structural dynamics that linear plate theory cannot be used to accurately predict structural responses at high excitation levels. In an attempt to overcome this weakness, several nonlinear plate models have been proposed. The nonlinearities introduced in the various models can be classified in two categories: geometric nonlinearity or material nonlinearity. The nonlinear model based on the geometric nonlinearity, also referred to as the large deflection model, has been extensively used in the literature. ${ }^{1-10}$ Although this model gives better predictions than those of the linear theory, it overestimates the frequency content or "broadening" of the response spectrum at high excitation levels.

To further improve the predictions of the nonlinear analysis, several damping models have been studied. Linear, nonlinear, and viscous damping are among the models used. ${ }^{11-15}$ Prasad and $\mathrm{Mei}^{16,17}$ used nonlinear damping in their large deflection model. A set of modal equations of the Duffing form coupled in the nonlinear stiffness and uncoupled in the nonlinear damping was derived. An approximate solution was obtained for rms quantities (such as displacement and strain) and spectral density functions by the equivalent linearization method. The nonlinear damping was found to contribute to the broadening of the power spectra at high excitation levels. However, Moyer ${ }^{18}$ and Reinhall and Miles ${ }^{19}$ showed that the method of equivalent linearization gives inaccurate response spectra for large deflections. The broadening of the response spectrum at high excitation levels was found to be caused by the nonlinear stiffness. ${ }^{19}$ Robinson and $\mathrm{Mei}^{20}$ studied the influence of nonlinear damping on panels random response using a time domain simulation. They found that the nonlinear damping was responsible for a narrowing of the nonlinear response power spectrum.

Recently, Robinson etal. ${ }^{21}$ used a finite element method to integrate the nonlinear plate equations with an equivalent linear viscous damping term to study the linear and nonlinear response of a plate subjected to a narrow band random excitation. Their results were in good agreement with experiments for low excitation levels. However, for high excitation levels, the model overpredicted the broadening of the response spectrum and predicted higher response levels at high frequencies.

In most structural dynamics analyses, there is little discussion about the effect of the surrounding acoustic fluid on the response of a structure to a given excitation field. Frendi, etal. ${ }^{22,23}$ showed that a strong coupling between plate vibration and the surrounding flow fields exists at high excitation levels.

In this paper, an attempt is made to explain the discrepancies between the experimental and numerical results obtained by Robinson, etal. ${ }^{21}$ Using the model developed by Frendi, etal. ${ }^{23}$ and changing the configuration of the computational domain to match that of the experiments, the response of a flexible plate to both a narrow band random excitation and a harmonic excitation is studied using two models. One model accounts for the full nonlinear coupling to the surrounding fluid. The other replaces the nonlinear coupling by 
an equivalent linear viscous damping on the plate.

The remainder of the paper is organized as follows. In section 2, a detailed description of the analytical model is given. Section 3 describes the numerical techniques, and the results are discussed in section 4 . Finally, the conclusions are given in section 5 .

\section{Formulation of the model}

As shown on fig. 1, the computational domain is composed of three regions. The acoustic fluid in the cavity region (or Top domain) is separated from that in the open space region (or Bottom domain) by a flexible plate clamped between two rigid plates. Acoustic disturbances are introduced at the left boundary of the Top domain and propagate over the rigid and flexible surfaces. There is no meanflow on either side of the flexible plate. The governing equations in the acoustic fluid regions are the two-dimensional, compressible, nonlinear Euler equations. In a cartesian coordinate system, $x$ and $y$, these equations can be written in conservation form as

$$
\frac{\partial Q}{\partial t}=\frac{\partial F}{\partial x}+\frac{\partial G}{\partial y}
$$

where $Q$ is the vector $(\rho, \rho u, \rho v, e)^{T}, \rho$ is the density, $\rho u$ and $\rho v$ are the $x$ and $y$ momenta respectively, and $e$ is the total energy per unit volume given by

$$
e=\frac{1}{2} \rho\left(u^{2}+v^{2}\right)+\rho c_{v} T .
$$

In eq. (1), the functions $F$ and $G$ are:

$$
F=\left(\begin{array}{c}
\rho u \\
\rho u^{2}+p \\
\rho u v \\
u(e+p)
\end{array}\right)
$$

and

$$
G=\left(\begin{array}{c}
\rho v \\
\rho u v \\
\rho v^{2}+p \\
v(e+p)
\end{array}\right) .
$$

In addition to Eq. (1), an ideal gas state equation is used

$$
p=\rho R T,
$$

where $p$ is the pressure, $\rho$ the density, $R$ the gas constant, and $T$ the temperature.

The equation describing the motion of the one-dimensional flexible surface is

$$
D \frac{\partial^{4} w}{\partial x^{4}}-N_{x} \frac{\partial^{2} w}{\partial x^{2}}+\rho_{p} h \frac{\partial^{2} w}{\partial t^{2}}+\left(\Gamma_{s}+\Gamma_{a}\right) \frac{\partial w}{\partial t}=\Delta p
$$


where $w$ is the plate transverse deflection, $\rho_{p}$ the mass per unit volume of the plate, and $h$ the plate thickness. The total viscous damping is the sum of the structural damping, $\Gamma_{s}$, and the acoustic damping, $\Gamma_{a}$. When the plate vibration is coupled to the surrounding acoustic fluid, $\Gamma_{a}=0$. In eq. (5), $D=E h^{3} / 12\left(1-\nu^{2}\right)$ is the stiffness of the plate, with $E$ being the modulus of elasticity and $\nu$ the Poisson ratio of the plate material. The coefficient $N_{x}$ in eq. (5) is given by

$$
N_{x}=\frac{E h}{2 L} \int_{x_{0}}^{x_{0}+L}\left(\frac{\partial w}{\partial x}\right)^{2} d x
$$

which represents the tension created by stretching of the plate due to bending. In eq. (6) $x_{0}$ is the origin of the flexible plate and $L$ its length. The forcing term on the right-hand-side of eq. (5) is

$$
\Delta p=p^{-}-p^{+}
$$

where $p^{+}$and $p^{-}$are the pressures on the surfaces above and below the plate, respectively. When acoustic coupling is neglected, the plate response is obtained by neglecting $p^{-}$in eq. (5) and using the same $p^{+}$at the plate center as that obtained by integration of the Euler equations. This is done in order to reproduce the results obtained by Robinson, etal. 21

Equations (1)-(7) are written in a nondimensional form using the following reference quantities for the different variables:

$$
\begin{gathered}
(x, y, w)_{\text {ref }}=l_{\text {ref }}, \quad t_{\text {ref }}=\frac{l_{\text {ref }}}{c_{\infty}}, \quad \text { and } \quad T_{\text {ref }}=\frac{c_{\infty}^{2}}{c_{v}} \\
\rho_{\text {ref }}=\rho_{\infty}, \quad\left(u, v, \frac{\partial w}{\partial t}\right)_{\text {ref }}=c_{\infty}, \quad \text { and } \quad(p, e)_{\text {ref }}=\rho_{\infty} c_{\infty}^{2} .
\end{gathered}
$$

The notation $(\cdot, \cdot)_{\text {ref }}$ is used to represent the reference quantity of the variables in parentheses. The various freestream fluid properties are those of air at sea level conditions which are: temperature $T_{\infty}=519^{\circ} \mathrm{R}$, density $\rho_{\infty}=1.147 \times 10^{-7} \mathrm{lb} \mathrm{sec}^{2} / \mathrm{in}^{4}$, pressure $p_{\infty}=14.7$ psi and sound speed $c_{\infty}=13392 \mathrm{in} / \mathrm{sec}$. The specific heat at constant volume is $c_{v}=6.1776 \times 10^{5}$ $\mathrm{in}^{2} /\left(\sec ^{2}{ }^{\circ} \mathrm{R}\right)$, the ratio of specific heats is $\gamma=c_{p} / c_{v}=1.4$, and the reference length is $l_{\text {ref }}=12.0 \mathrm{in}$.

\section{Method of solution}

The unsteady Euler equations (eq. (1)) are solved using an explicit finite difference scheme. The scheme, which is a generalization of MacCormack's scheme obtained by Gottlieb and Turkel, ${ }^{24}$ is fourth order accurate in space and second order accurate in time. The numerical scheme, applied to a one-dimensional equation of the form

$$
\frac{\partial u}{\partial t}=\frac{\partial F}{\partial x}
$$

consists of a predictor step given by

$$
u_{i}^{\star}=u_{i}^{n}+\frac{\Delta t}{6 \Delta x}\left(-7 F_{i}+8 F_{i+1}-F_{i+2}\right)
$$


followed by a corrector step of the form

$$
u_{i}^{n+1}=\frac{1}{2}\left[u_{i}^{\star}+u_{i}^{n}+\frac{\Delta t}{6 \Delta x}\left(7 F_{i}^{\star}-8 F_{i-1}^{\star}+F_{i-2}^{\star}\right)\right] .
$$

In the above equations, the subscript $i$ denotes the spatial grid point and the superscript $n$ the time level. The fourth-order accuracy is obtained by alternating the scheme given above with its symmetric variant. ${ }^{24}$ Operator splitting is used to reduce the two-dimensional problem to a sequence of one-dimensional problems. If $L_{x}$ and $L_{y}$ denote the solution operators for the one-dimensional $x$ and $y$ problems, then the solution to eq. (1) is obtained by

$$
Q^{n+2}=L_{x} L_{y} L_{y} L_{x} Q^{n}
$$

Further details regarding the method and the advantage of fourth order schemes can be found in Bayliss, etal. ${ }^{25}$

The boundary conditions employed on the rigid and flexible surfaces for the Euler equations are

$$
v=0 \quad \text { and } \quad T=T_{w}
$$

over the rigid surfaces, and

$$
v=\frac{\partial w}{\partial t} \quad \text { and } \quad T=T_{w}
$$

over the flexible surface. In eqs. (13) and (14) $T_{w}$ is a specified wall temperature; in this paper it is taken to be the free-stream temperature, $T_{\infty}$. The $x$-component of the velocity $(u)$ is obtained through linear extrapolation from the interior over both the rigid and flexible surfaces.

The pressure boundary conditions are as follows: over the rigid surfaces where the time rate of change of the normal momentum is zero, the pressure is calculated using the normal momentum equation by simply imposing the normal gradient of the sum of pressure and vertical momentum flux to be zero $\left(\frac{\partial}{\partial y}\left(p+\rho v^{2}\right)=0\right)$. Over the flexible part of the surface, a linear extrapolation from the interior is used. At the left boundary of the Top domain, a nondimensional perturbation velocity is specified $\tilde{u}$ as

$$
\bar{u}=\epsilon R(t) \quad \text { or } \quad \tilde{u}=\epsilon \sin (\omega t),
$$

where $R(t)$ represents a narrow-band random excitation and $\tilde{u}$ is a nondimensional perturbation velocity. In eq. (15), $\epsilon$ is the peak amplitude of the disturbance for the harmonic case (with $\omega$ being the frequency), and an rms amplitude in the random case. Using the routine RNNOF of the IMSL library ${ }^{26}$, Gaussian random data is generated; then, using a quadratic narrow-band filter, the data is filtered to give the desired input. The pressure and vertical velocity $(v)$ are obtained by linear interpolation from the interior, and the temperature is imposed to be constant $\left(T=T_{w}\right)$. The remaining non-physical boundary conditions (Top 
domain right boundary, Bottom domain right, left, and bottom boundaries) are derived using the method of characteristics. ${ }^{27}$

The plate equation is integrated using an implicit finite difference method for structural dynamics developed by Hoff and Pahl. ${ }^{28}$ The calculation of $N_{x}$ was done using Simpson's rule of integration. The boundary conditions used to solve the plate equation are those for a clamped plate

$$
w=\frac{\partial w}{\partial x}=0 \quad \text { at } \quad x=x_{0}, x_{0}+L .
$$

The coupling between the acoustic fluid and the flexible plate is performed as follows. The method used in the fluid is explicit. Thus the pressure fields in the Top and Bottom domains are updated by using the value of $\frac{\partial w}{\partial t}$ at the previous time-step as a boundary condition for the Euler equations. Then, using the new values of $p^{+}$and $p^{-}$, the plate equation is integrated to obtain the new vertical velocity $\frac{\partial w}{\partial t}$. This procedure is repeated at every time-step.

For every coupled calculation, the time history of the pressure at the center of the flexible plate in the Top domain is stored in order to be used as input for the equivalent uncoupled case. This was done in order to match the numerical procedure used by Robinson, etal. $^{21}$

\section{Results and Discussion}

The results presented in this paper are obtained for a flexible plate having the following properties: stiffness $D=1095.6 \mathrm{lbf} \cdot$ in, mass per unit area $\rho_{p} h=2.21 \times 10^{-5} \mathrm{lbf}$ $\cdot \sec ^{2} / \mathrm{in}^{3}$, and a Poisson ratio $\nu=0.3$. Two values of total viscous damping $\left(\Gamma_{s}+\Gamma_{a}\right)$ are used; $2.5 \times 10^{-5} \mathrm{lbf} \cdot \mathrm{sec} / \mathrm{in}^{3}$ used in the fully coupled calculation (where $\Gamma_{a}=0$ ) and $5 \times 10^{-4} \mathrm{lbf} \cdot \mathrm{sec} / \mathrm{in}^{3}$ used in the uncoupled calculation. These two values are chosen such that the two calculations predict the same response for low-intensity excitations (linear vibration regime). The plate is 15 in long, 11 in wide, and 0.13 in thick. The first natural frequency of the plate is $112 \mathrm{~Hz}$. The Top domain of Fig. 1 is 12 in high in the y-direction and 120 in long in the $x$-direction, and the number of computational points used are 101 and 181 in both directions, respectively. The Bottom domain is 120 in long in both directions with 161 points used in each direction.

The power spectra shown here are for the center plate displacement response and the pressure on either side of the plate center. Also typical instantaneous pressure distributions are shown for both the Top and Bottom domains.

\section{- Previous Experimental and Numerical Results}

At first the results obtained in Ref. 21 are summarized. Figure 2 shows the power spectral density of the strain obtained both experimentally and numerically for a narrowband excitation sound pressure level of $140 \mathrm{~dB}$. The figure shows that the power spectrum obtained from the simulation is in good agreement with that given by the experiment near the fundamental frequency $(112 \mathrm{~Hz})$ where the measured strains are within the sensitive range of the strain gages. Away from the fundamental frequency, the measured strains are 
small compared to the sensitivity, which is approximately $1.0 \mu$-strain squared per $\mathrm{Hz}$, and therefore not very accurate. As the sound pressure level is increased to $160 \mathrm{~dB}$, figure 3 , the simulation overpredicts the broadening of the spectrum and the response at high frequencies. It is important to restate that an equivalent linear viscous damping was used in the plate model, and that the nonlinear coupling to the surrounding acoustic fluid was neglected.

\section{- Current Model: (1) Random Excitation}

In order to obtain a pressure input spectrum similar to that of the experiments described above, the random data generated by the IMSL routine RNNOF is filtered using a quadratic narrow-band filter of bandwidth 50 to $500 \mathrm{~Hz}$. Figure 4 shows that both the fully coupled and uncoupled models predict the same displacement frequency response of the plate for a low-intensity noise having a maximum sound pressure level of $120 \mathrm{~dB}$. The spectrum obtained by the uncoupled model shows a more pronounced peak at $560 \mathrm{~Hz}$, which corresponds to a natural frequency of the plate. The plate response is linear and is dominated by the first mode. Due to the high cost in CPU time of the coupled calculations, the time histories of the various quantities were not long enough to give smoother power spectra.

Increasing the noise intensity to a maximum sound pressure level of $140 \mathrm{~dB}$ leads to a nonlinear plate response. The frequency content of the response shifts toward higher frequencies and the spectrum broadens, as shown by fig. 5 . However, the shift and the broadening are different for the two models. The power spectrum predicted by the uncoupled model shows a greater shift toward high frequencies and more broadening than that predicted by the coupled model. The plate response at high frequencies is also higher when the uncoupled model is used. The difference between the coupled and uncoupled power spectra is similar to that observed earlier between experiments and simulation (see fig. 3 ). This result indicates that acoustic coupling at high sound pressure levels is important for the accurate prediction of the plate response.

Figure 6 shows the power spectra of the pressure on both sides of the plate center. Notice that the pressure on the top surface is dominated by the input pressure while the pressure on the bottom surface is due to the vibration of the plate and has, therefore, a power spectrum similar to that of the response. An instantaneous pressure distribution in the Top domain is shown in fig. 7. Since the input is random, several peaks are observed in the pressure field superimposed on a strong low frequency. The instantaneous pressure distribution in the Bottom domain shows the presence of one dominant frequency corresponding to that of the plate response, fig. 8.

\section{- Current Model: (2) Harmonic Excitation}

For an excitation frequency $f=112 \mathrm{~Hz}$ (corresponding to the first mode of the plate) and an amplitude of $150 \mathrm{~dB}$, the plate response is nonlinear, as shown by fig. 9. The power spectra of the center plate displacement show several harmonics and subharmonics characteristic of the nonlinear response. For this excitation level, both the fully coupled and uncoupled models predict the same response power spectrum (similar results are obtained for lower $\mathrm{dB}$ levels). This result is attributed to the weak nonlinearity in the radiation field. Figure 10 shows the power spectra of the pressure on both sides of the plate center. The 
power spectrum of the pressure on the top surface shows a strong peak at the fundamental frequency $(112 \mathrm{~Hz})$ and negligible harmonic content, indicative of weak nonlinearity. The power spectrum of the radiated pressure on the bottom surface more closely resembles to the response power spectrum with slightly stronger harmonics as should be expected, since the coupling between the plate vibration and the acoustic fluid is obtained through the vertical velocity. Due to transmission loss, the level of the radiated pressure on the bottom surface is $20 \mathrm{~dB}$ lower than that of the top surface. The weak nonlinearity in the pressure field is further evidenced by fig. 11; where the Top domain power spectra of the pressures at the inflow and near the center of the flexible plate are shown. The two spectra are nearly identical except for a negligible difference in the levels of the harmonics indicating a weak nonlinearity in addition to the contribution of the flexible plate.

Increasing the level of the excitation source to $165 \mathrm{~dB}$ leads to a stronger contribution of the harmonics to the plate response and much less contribution from the subharmonics, fig. 12. This result is in agreement with earlier work ${ }^{23}$ which showed that the window to complex dynamics is very narrow and that an increase in excitation level can lead to a more stable system. The two models do not predict the same displacement power spectrum of the plate. At high frequencies, the uncoupled model predicts a higher level of the response peaks. This is similar to the random excitation case, except that the peaks are not shifted. Figure 13 shows that the pressure fields on both sides of the plate are nonlinear. Both pressure power spectra show a strong harmonic content. The nonlinearity in the Top domain pressure field is shown in fig. 14. The levels of the harmonics on the flexible plate are higher due to both the plate vibrations and the nonlinear wave propagation. The latter point can be seen on the figure through the change in magnitude of the peak at $560 \mathrm{~Hz}$ and its harmonic $1120 \mathrm{~Hz}$. On the flexible plate, the $560 \mathrm{~Hz}$ peak (which corresponds to a plate mode) is higher than its harmonic $(1120 \mathrm{~Hz})$. However, at the inflow the two peaks are nearly the same. This indicates that while the $560 \mathrm{~Hz}$ peak has decayed significantly its harmonic has decayed only slightly. This is a characteristic of nonlinear wave propagation. Similar results are obtained for the Bottom domain pressure field.

\section{Conclusions}

Based on the results obtained in this paper, the following conclusions can be made:

(1) The coupling between the acoustic fluid with no meanflow and the plate vibration is not important at low excitation levels for both random and harmonic excitations, as was reported by Frendi, etal. 23

(2) The acoustic coupling is important for accurate prediction of the plate response at high excitation levels. The present results show that the absence of acoustic coupling in earlier models may be the reason for the discrepancy between numerical and experimental results. When acoustic coupling is accounted for, less spectral broadening and frequency shift is obtained. The plate response is lower at higher frequencies, which is in agreement with experimental observations. ${ }^{21}$

(3) In the harmonic case, no shift in the peaks of the response is obtained; however, 
the uncoupled model predicts higher levels of the higher harmonics. The results also show that the coupling between the acoustic fluid and the plate vibration is important only when both the structural response and the acoustic radiation are nonlinear, which is in agreement with previous results. ${ }^{23}$

\section{Ackowledgements}

The first author was supported by NASA Langley Research Center under contract NAS1-19317.

\section{References}

1 Tseng, W. Y. and Dugundji, J., "Nonlinear Vibrations of a Beam Under Harmonic Excitation," JournalofAppliedMechanics, Vol. 37, No. 2, 1970, pp. 292-297.

2 Srinivasan, A. V., "Nonlinear Vibrations of Beams and Plates," International JournalofNonlinearMechanics, Vol. 1, 1966, pp. 179-191.

${ }^{3}$ Maestrello, L., Frendi, A., and Brown, D. E., "Nonlinear Vibration and Radiation From a Panel with Transition to Chaos Induced by Acoustic Waves," AIAAJournal, in press, 1992.

4 Yamaki, N., and Mori, A., "Nonlinear Vibrations of a Clamped Beam with Initial Deflection and Initial Axial Displacement, Part I: Theory," JournalofSoundandVibration, Vol. 71, No. 3, 1980, pp. 333-346.

5 Yamaki, N., Otomo, K., and Mori, A., "Nonlinear Vibrations of a Clamped Beam with Initial Deflection and Initial Axial Displacement, Part II: Experiment," Journal ofSoundandVibration, Vol. 71, No. 3, 1980, pp. 333-346.

${ }^{6}$ Ibrahim, R. A., and Roberts, J. W., 1976. "Broad-Band Random Excitation of a Two Degree-of-Freedom System with Autoparametric Coupling," JournalofSoundand Vibration, Vol. 44, No. 3, 1976, pp. 335-348.

${ }^{7}$ Mei, C. and Decha-Umphai, K., "A Finite Element Method for Nonlinear Forced Vibrations of Beams," JournalofSoundandVibration, Vol. 102, No. 3, 1985, pp. 369-380.

8 Chia, C. Y., Nonlinear Analysis of Plates, McGraw-Hill, New York,1980, pp. $1-51$.

9 Dowell, E. H., "Flutter of a Buckled Plate as and Example of Chaotic Motion of a Deterministic Autonomous System," JournalofSoundandVibration, Vol. 85, No. 3, 1982, pp. 333-344.

10 Dowell, E. H., and Pezeshki, C., "On the Understanding of Chaos in Duffings Equation Including a Comparison with Experiment," JournalofAppliedMechanics, Vol. 53, No. 1, 1986, pp. 5-9.

${ }^{11}$ Reed, R. R., "Analysis of Structural Response with Different Forms of Damping," NASA TND-3861, 1967.

12 Jacobsen, L. S., "Steady State Vibration as Influenced by Damping, Part I," Transactionofthe ASME, Vol. 52, 1930, pp. 169-181.

13 Torres, M. R., and Mote Jr., C. D., "Expected Equivalent Damping Under Random Excitation," JournalofEngineeringfor Industry Transactionofthe ASME, Vol. 91, 1969, pp. 967-974. 
14 Tang, D. M., and Dowell, E. H., "Random Response of Beams and Plates with Slipping at the Support Boundaries," AIAA/ASME/ASCE/AHS 27th Structures, Structural Dynamics and Materials Conference, San Antonio, Texas, 1986.

15 Dowell, E. H., "Damping in Beams and Plates Due to Slipping at the Support Boundaries," JournalofSoundandVibration, Vol. 105, No. 2, 1986, pp. 243-253.

${ }^{16} \mathrm{Mei}, \mathrm{C}$., and Prasad, C. B., "Effects of Non-linear Damping on Random Response of Beams to Acoustic Loading," JournalofSoundandVibration, Vol. 117, No. 1, 1987, pp. 173-186.

17 Prasad, C. B., "The effects of Nonlinear Damping on the Large Deflection Response of Structures Subjected to Random Excitation," PhD Thesis, Mechanical Engineering Department, Old Dominion University, Norfolk, Virginia, 1987.

18 Moyer, E. T. Jr., "Time Domain Simulation of the Response of Geometrically Nonlinear Panels Subjected to Random Loading," AIAA/ASME/ASCE/AHS 29th Structures, Structural Dynamics and Materials Conference, 1988, pp. 210-218.

${ }^{19}$ Reinhall, P. G., and Miles, R. N., 1989. "Effect of Damping and Stiffness on the Random Vibration of Non-Linear Periodic Plates," JournalofSoundandVibration, Vol. 131, No. 1, 1989, pp. 33-42.

${ }^{20}$ Robinson, J., and Mei, C., "The Influence of Nonlinear Damping on the Random Response of Panels by Time Domain Simulation," 12th AIAA Aeroacoustics Meeting, 1989, Paper 89-1104.

${ }^{21}$ Robinson, J., Rizzi, S., Clevenson, S., and Daniels, E., "Large Deflection Random Response of Flat and Blade Stiffened Carbon-Carbon Panels," AIAA-92-2390, presented at the 33rd Annual Meeting on Structures, Structural Dynamics and Materials Conference organized by AIAA-ASME-ASCE-AHS-ASC, 1992.

22 Frendi, A., Maestrello, L., and Bayliss, A., "On the Coupling Between a Supersonic Laminar Boundary Layer and a Flexible Surface," ICASE Report 92-42, Accepted for publication in the AIAAJournal, 1992.

${ }^{23}$ Frendi, A., Maestrello, L., and Bayliss, A., "Coupling Between Plate Vibration and Acoustic Radiation," To be presented at the AIAA 31st Aerospace Sciences Meeting and Exhibit, Reno, Nevada, January 1993.

24 Gottlieb, D., and Turkel, E., "Dissipative Two-Four Methods for Time Dependent Problems," MathematicsofComputation, Vol. 30, 1976, pp. 703-723.

${ }^{25}$ Bayliss, A., Parikh, P., Maestrello, L., and Turkel, E., "A Fourth Order Scheme for the Unsteady Compressible Navier-Stokes Equations," AIAA-85-1694, 1985.

${ }^{26}$ IMSL Statistical Library, Version 2.0, Volume 3, p. 1317, Houston, Texas, 1991.

27 Abarbanel, S. S., Don, W. S., Gottlieb, D., Rudy, D. H., and Townsend, J. C., "Secondary Frequencies in the Wake of a Circular Cylinder with Vortex Shedding," ICASE Report 90-16, 1990.

${ }^{28}$ Hoff, C., and Pahl, P. J., "Development of an Implicit Method With Numerical Dissipation From a Generalized Single-Step Algorithm for Structural Dynamics," Computa, tionalMethodsin AppliedMechanicalEngineering, Vol. 67, 1988, pp. 367-385. 


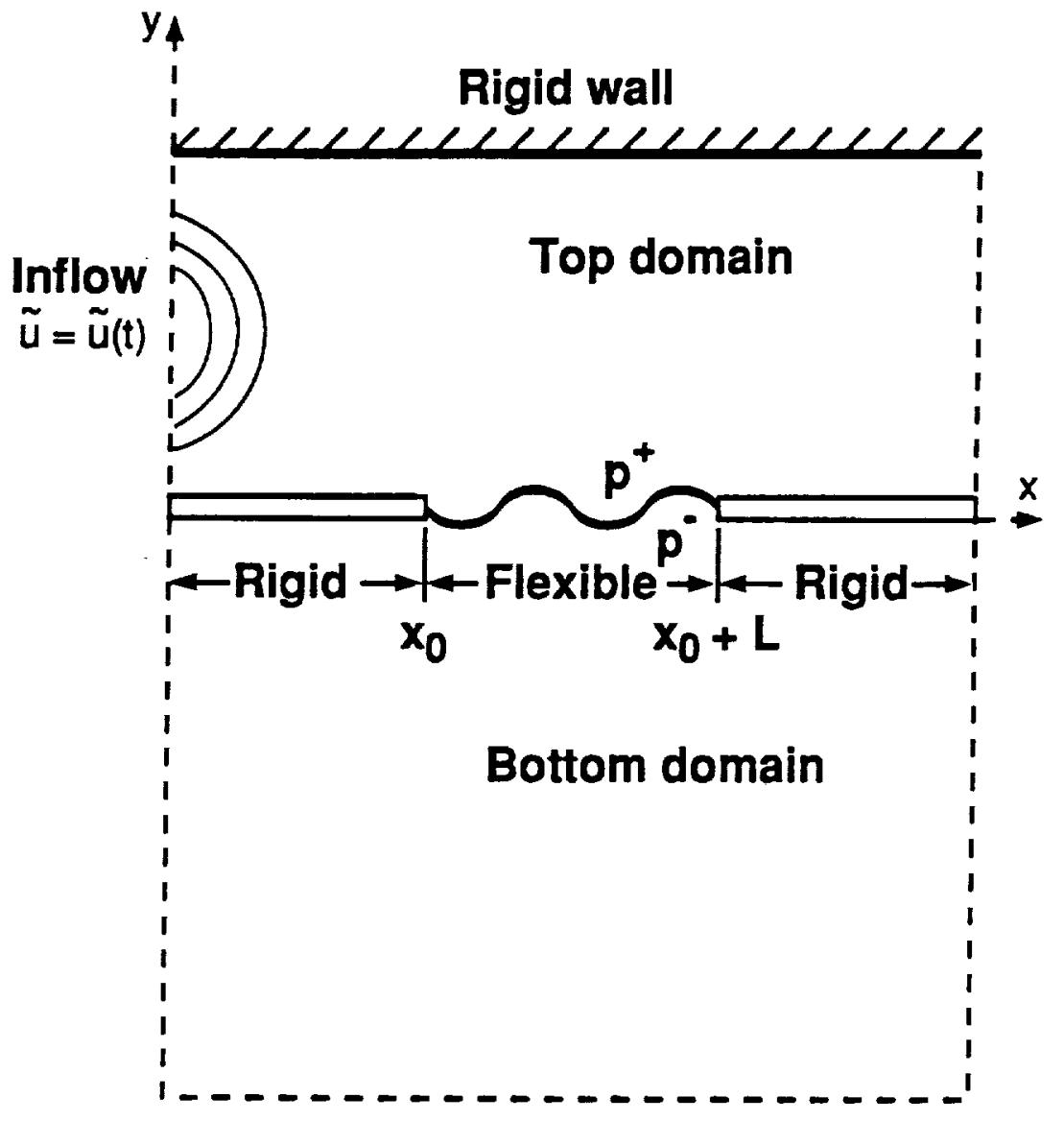

Figure 1: Computational Domain 


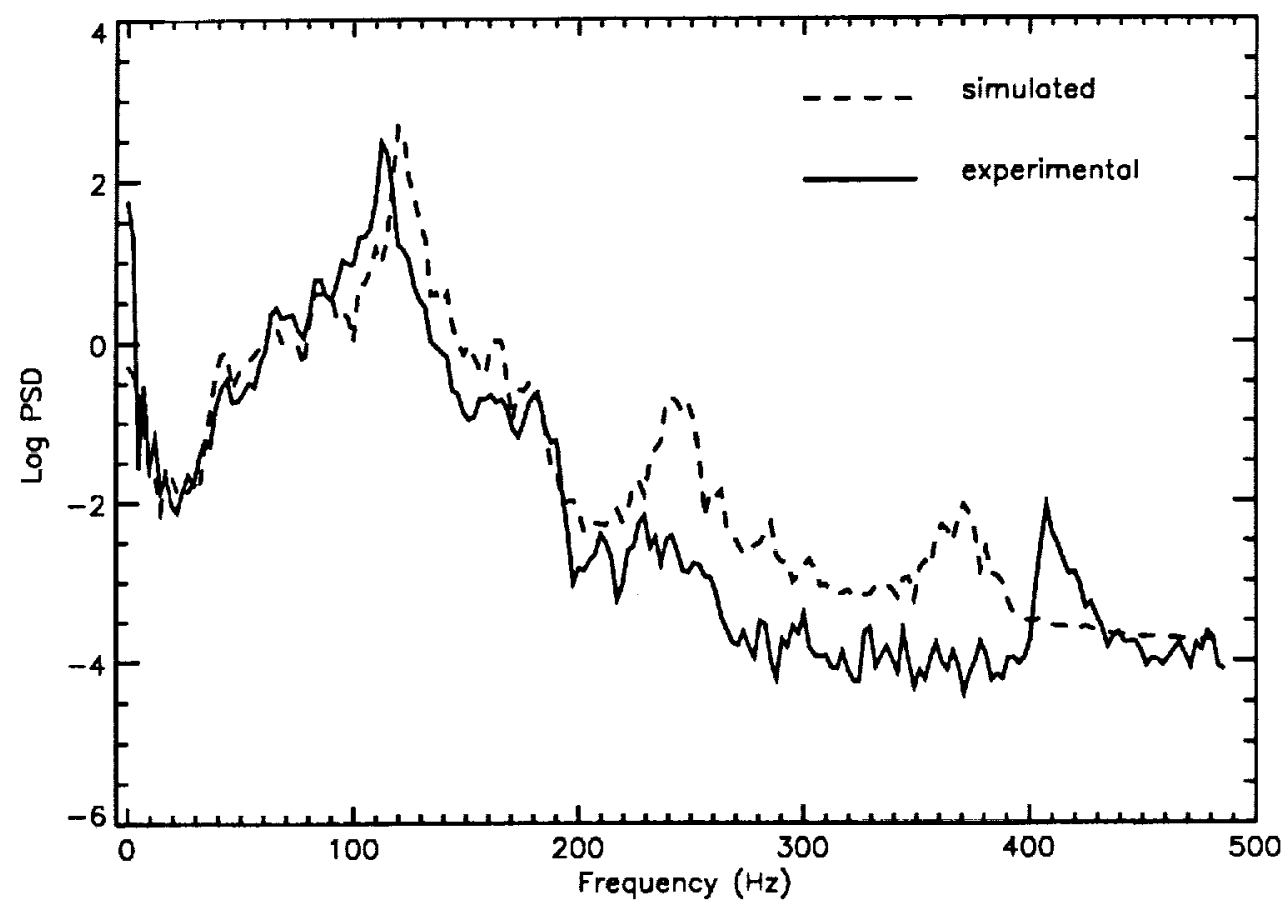

Fig. 2: Experimental and simulated power spectra of the strain for an overall sound pressure level of $140 \mathrm{~dB}$. (Robinson, etal. ${ }^{21}$ )

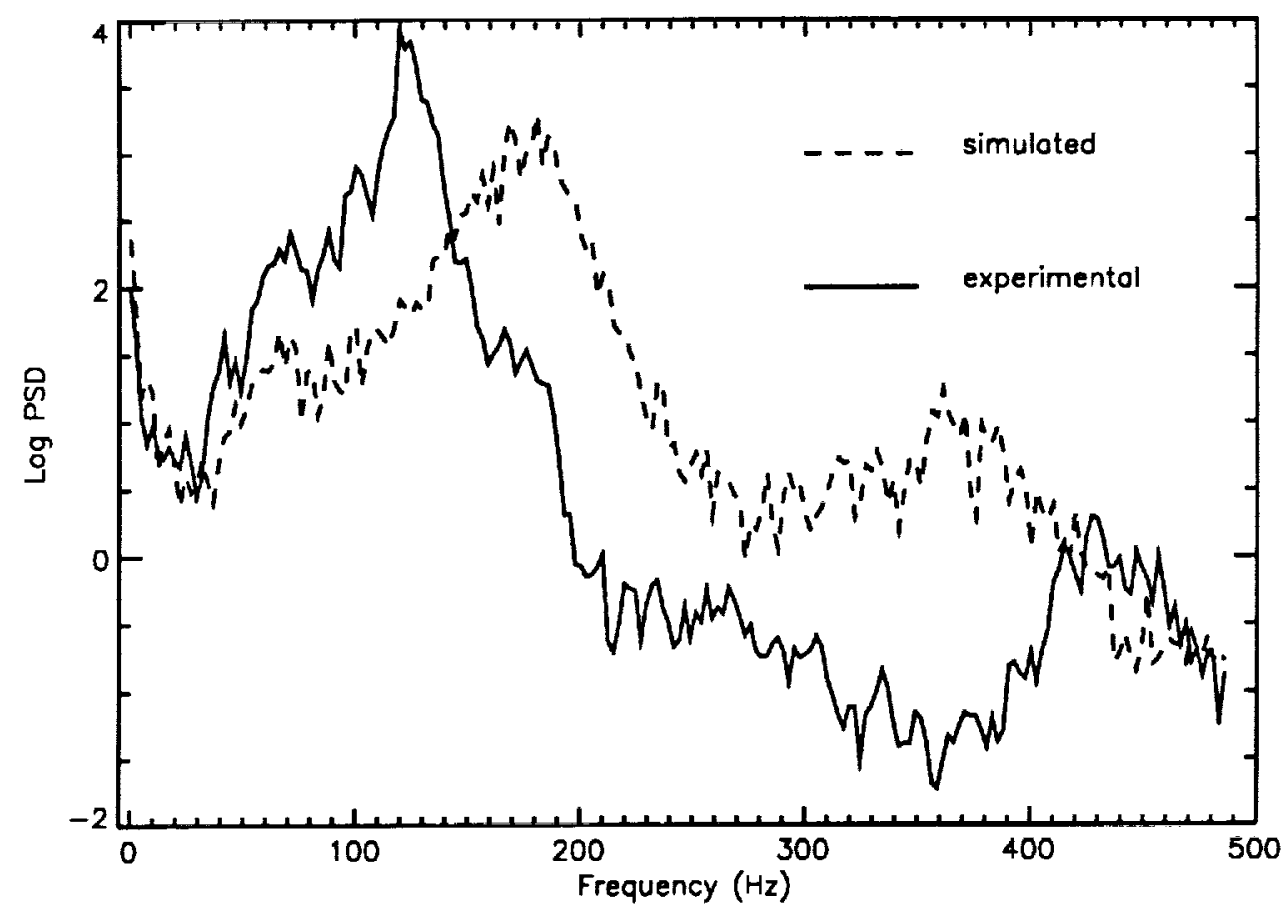

Fig. 3: Experimental and simulated power spectra of the strain for an overall sound pressure level of $160 \mathrm{~dB}$. (Robinson, etal. ${ }^{21}$ ) 


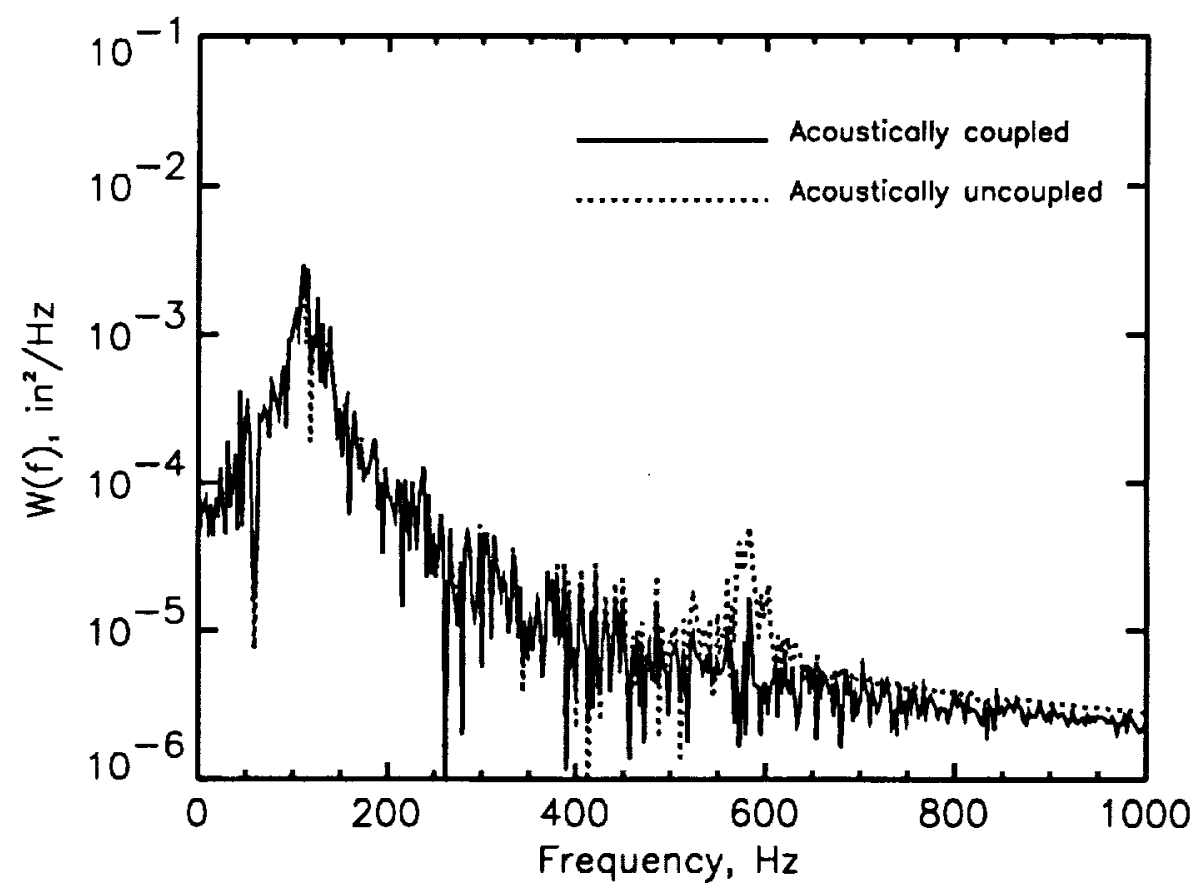

Fig. 4: Comparison of the power spectra of the center plate displacement obtained by the coupled and uncoupled models for a maximum random excitation level of $120 \mathrm{~dB}$.

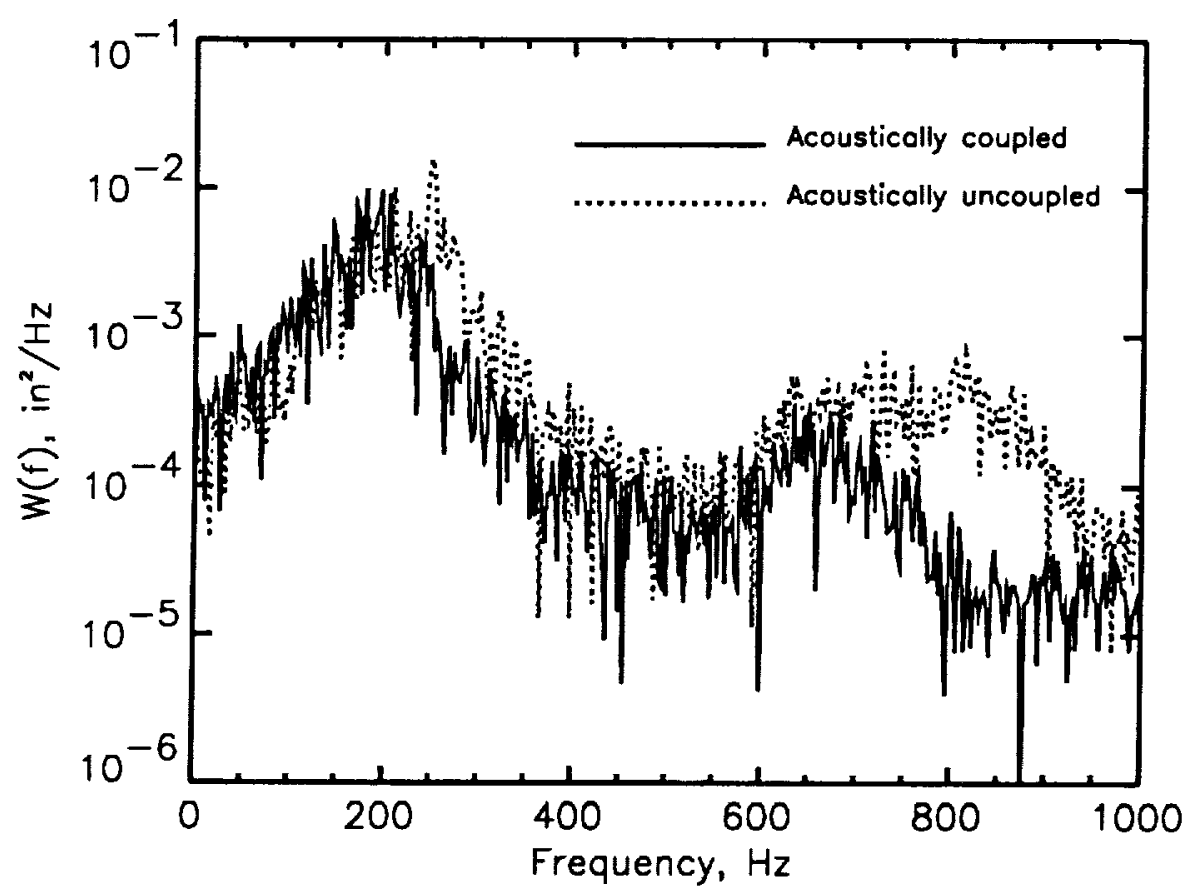

Fig. 5: Comparison of the power spectra of the center plate displacement obtained by the coupled and uncoupled models for a maximum random excitation level of $140 \mathrm{~dB}$. 


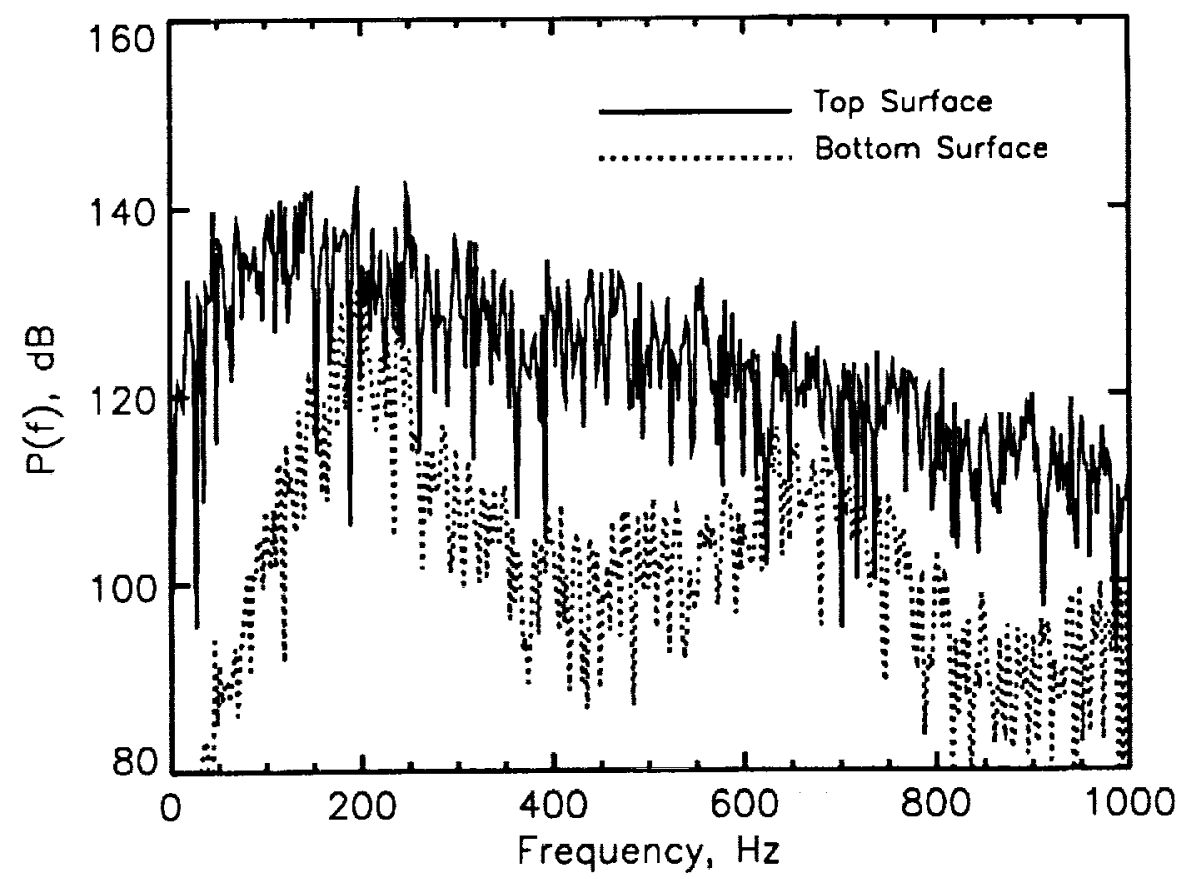

Fig. 6: Power spectra of the surface pressure on both sides of the plate center.

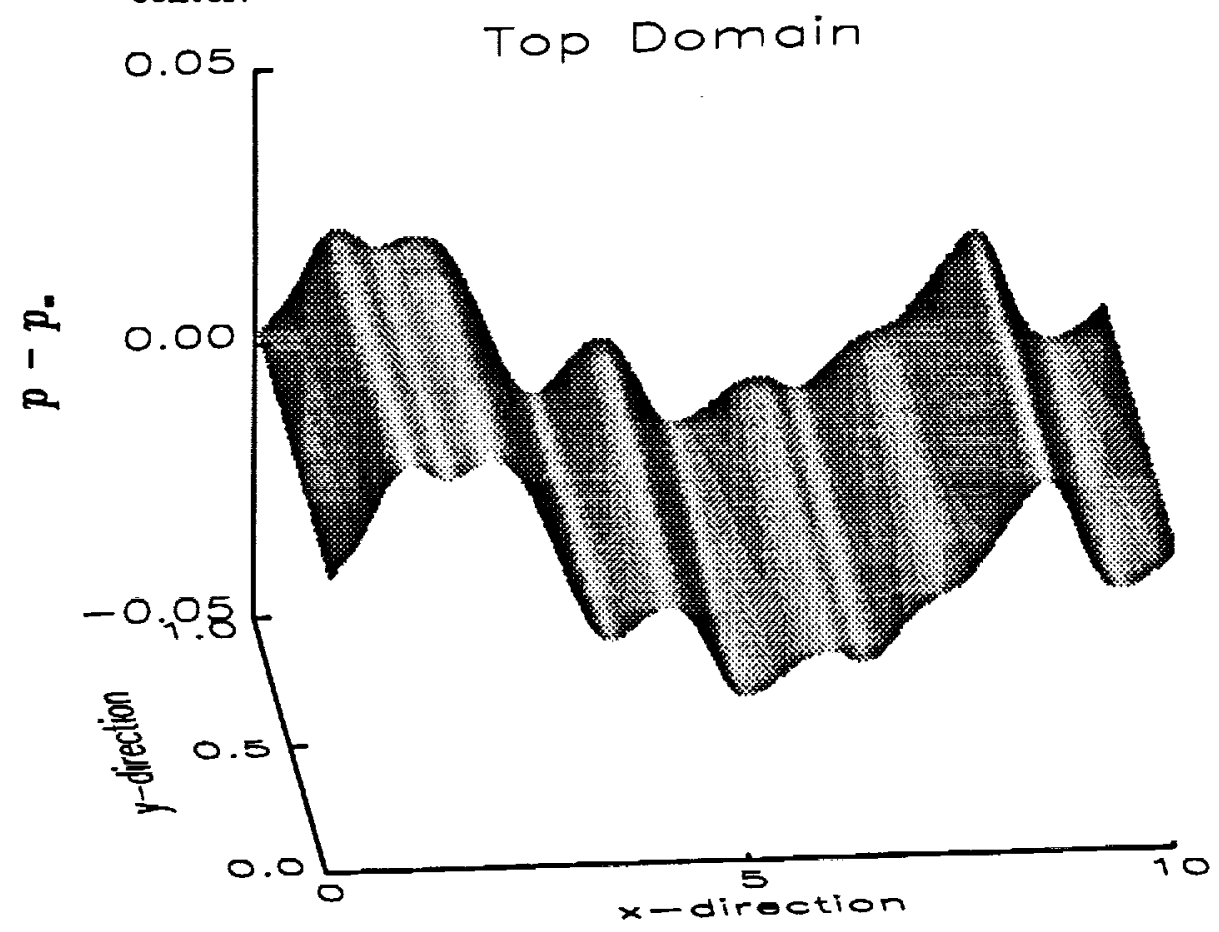

Fig. 7: Instantaneous pressure distribution in the Top domain for a maximum random excitation of $140 \mathrm{~dB}$. 


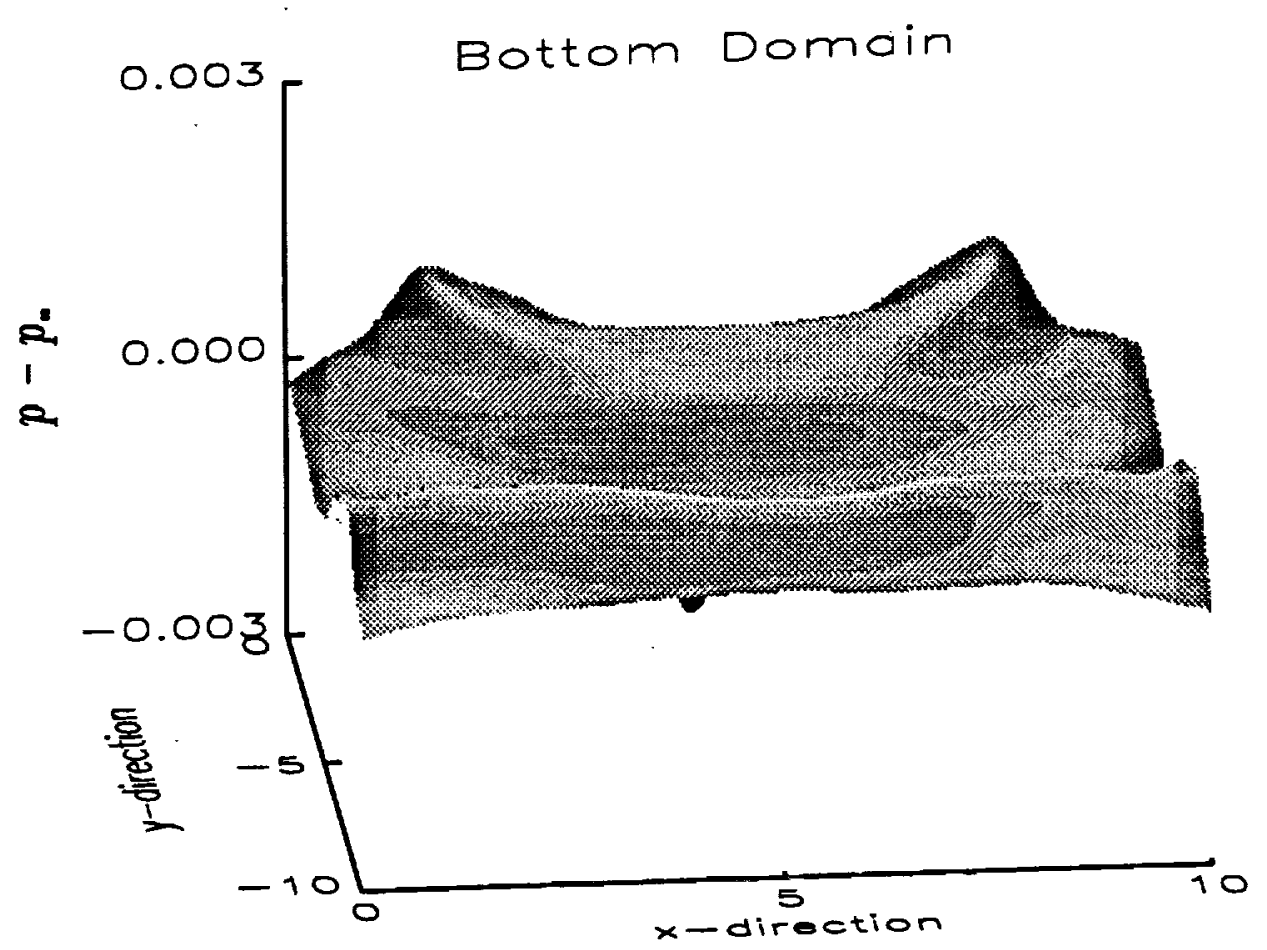

Fig. 8: Instantaneous pressure distribution in the Bottom domain for a maximum random excitation of $140 \mathrm{~dB}$.

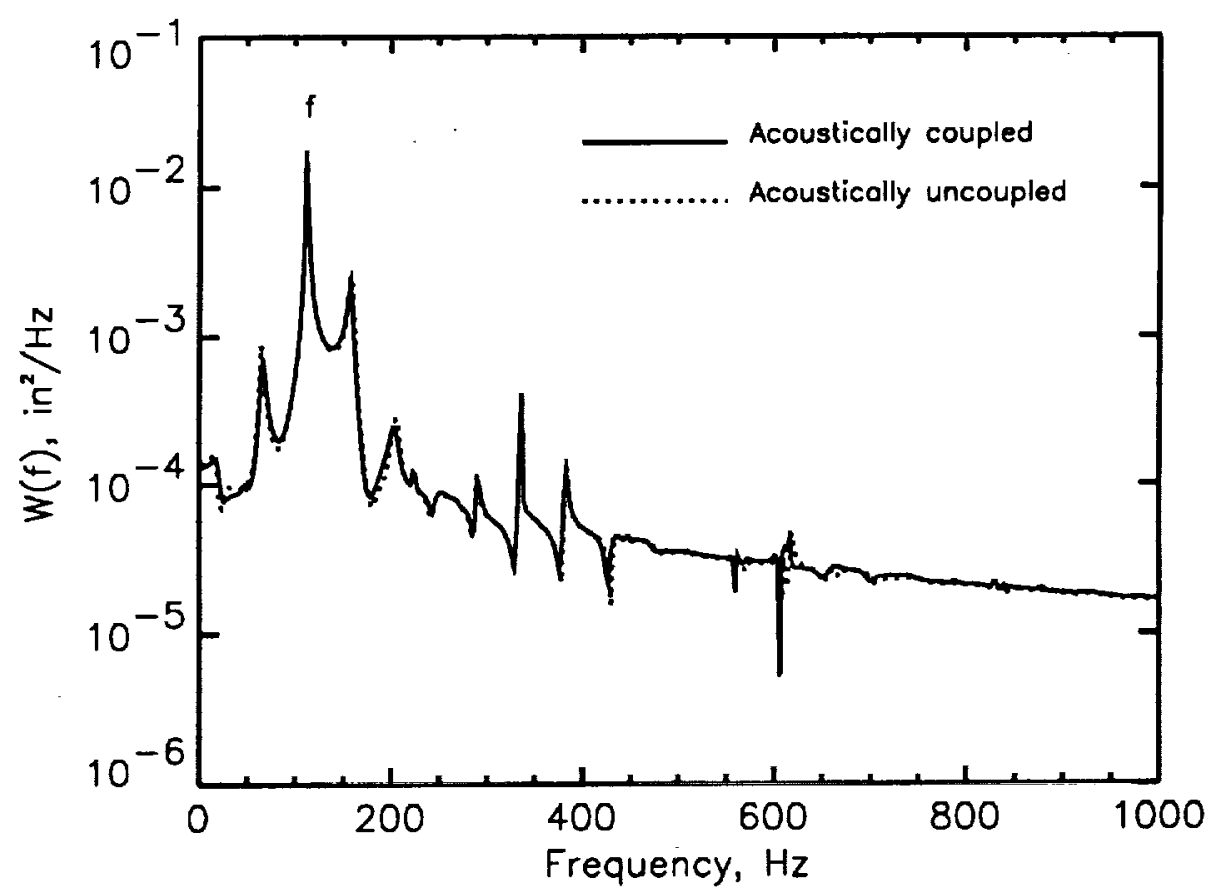

Fig. 9: Comparison of the power spectra of the center plate displacement obtained by the coupled and uncoupled models for harmonic excitation with a frequency of $112 \mathrm{~Hz}$ and a peak amplitude of $150 \mathrm{~dB}$. 


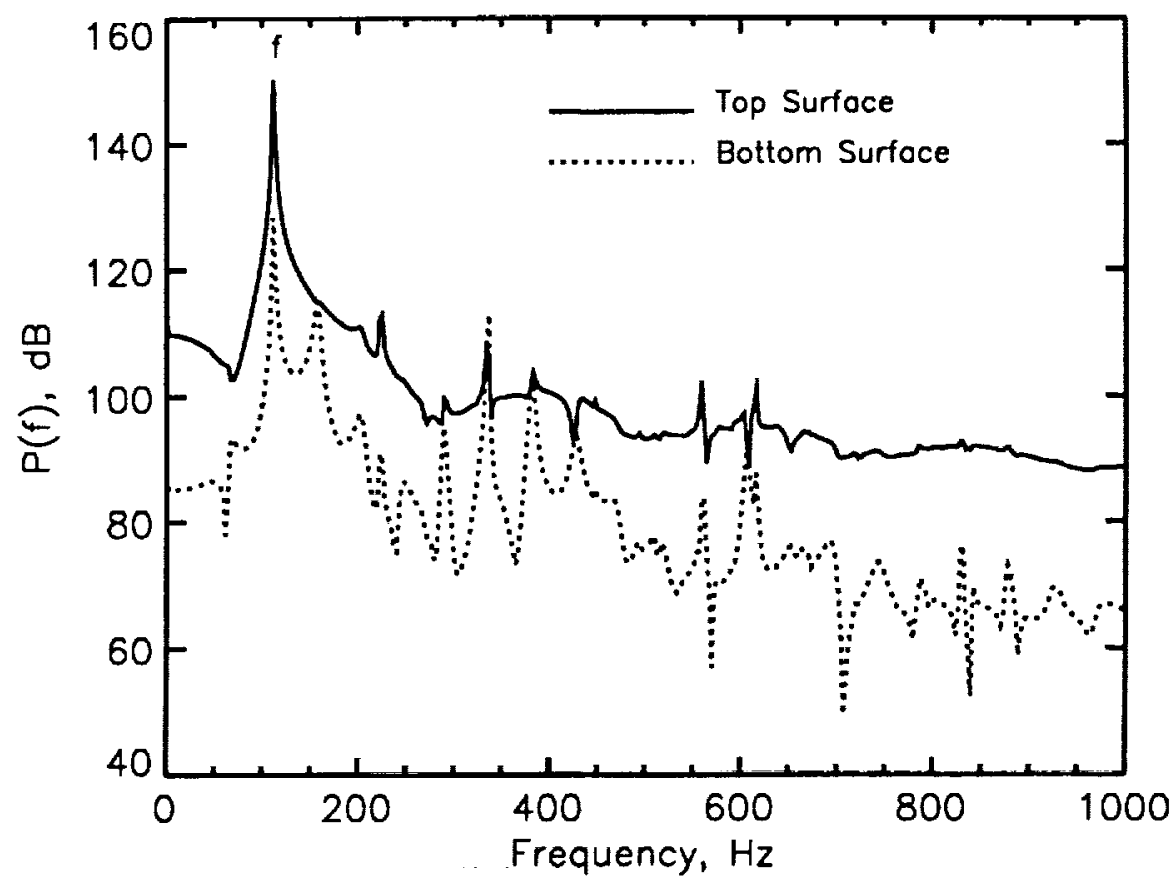

Fig. 10: Power spectra of the surface pressure on both sides of the plate center.

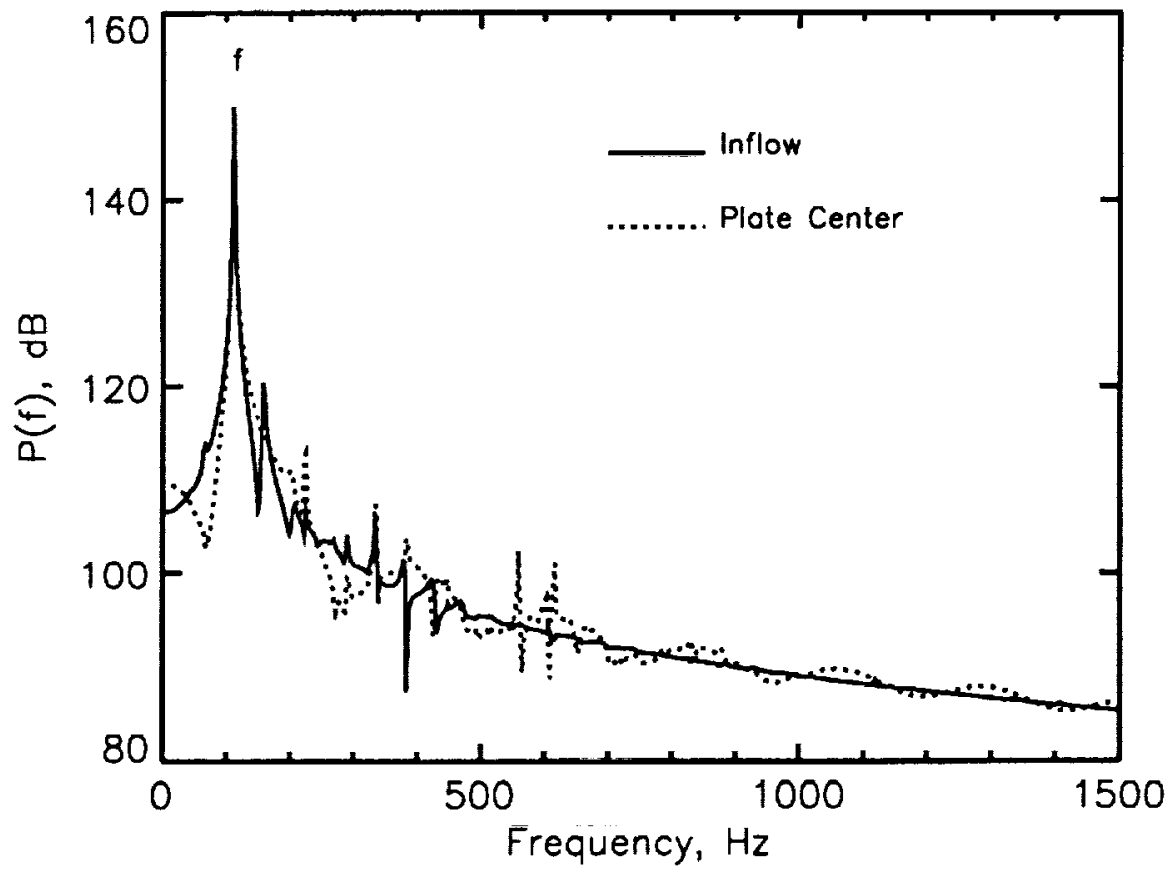

Fig. 11: Power spectra of the Top domain pressures at the inflow and near the center of the flexible plate. 


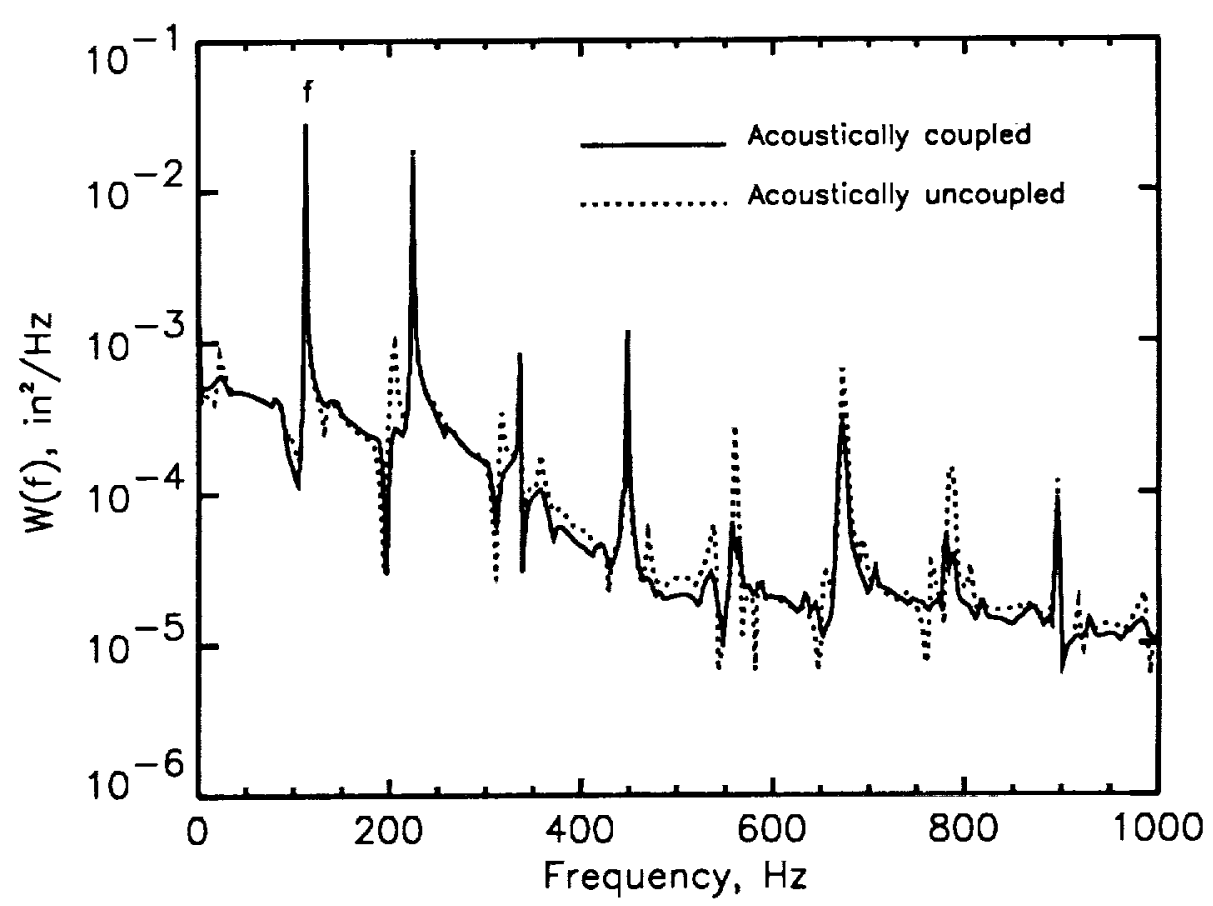

Fig. 12: Comparison of the power spectra of the center plate displacement obtained by the coupled and uncoupled models for harmonic excitation with a frequency of $112 \mathrm{~Hz}$ and a peak amplitude of $165 \mathrm{~dB}$.

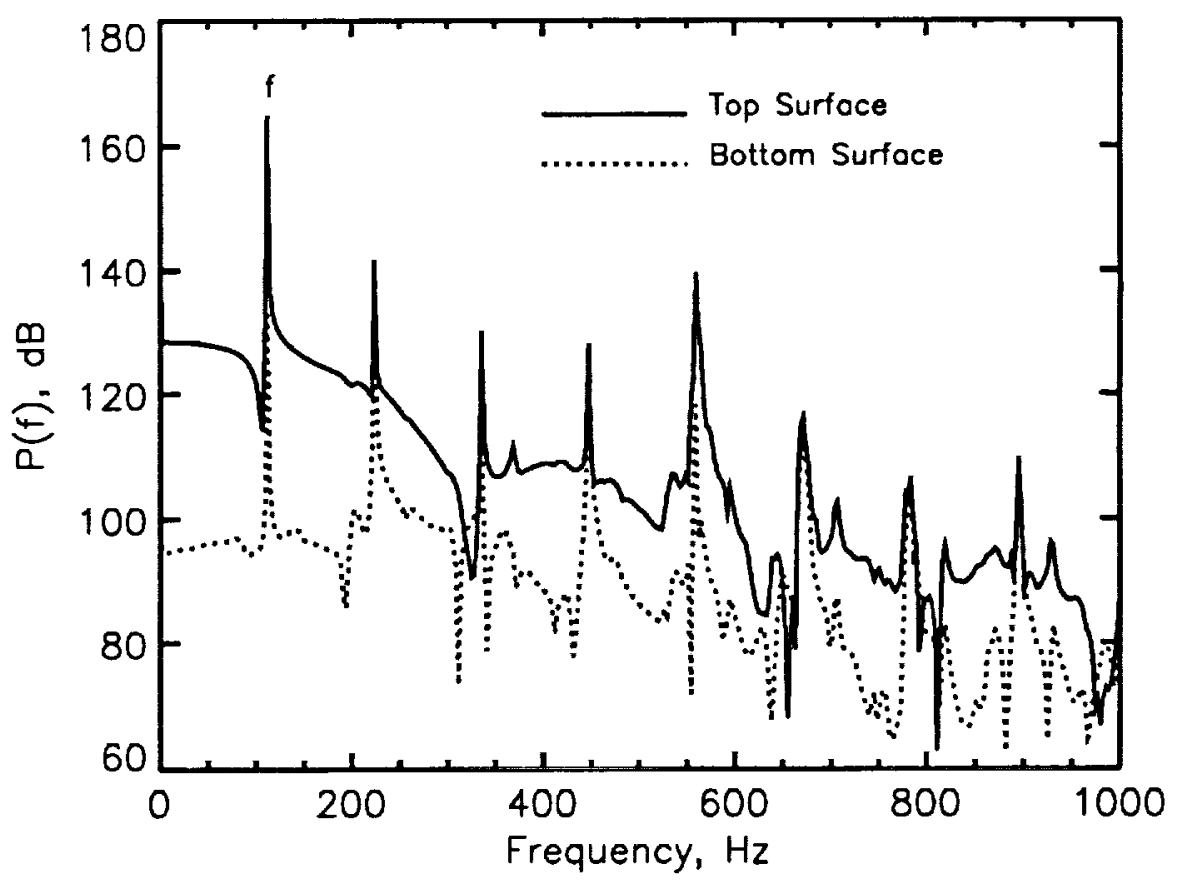

Fig. 13: Power spectra of the surface pressure on both sides of the plate center. 


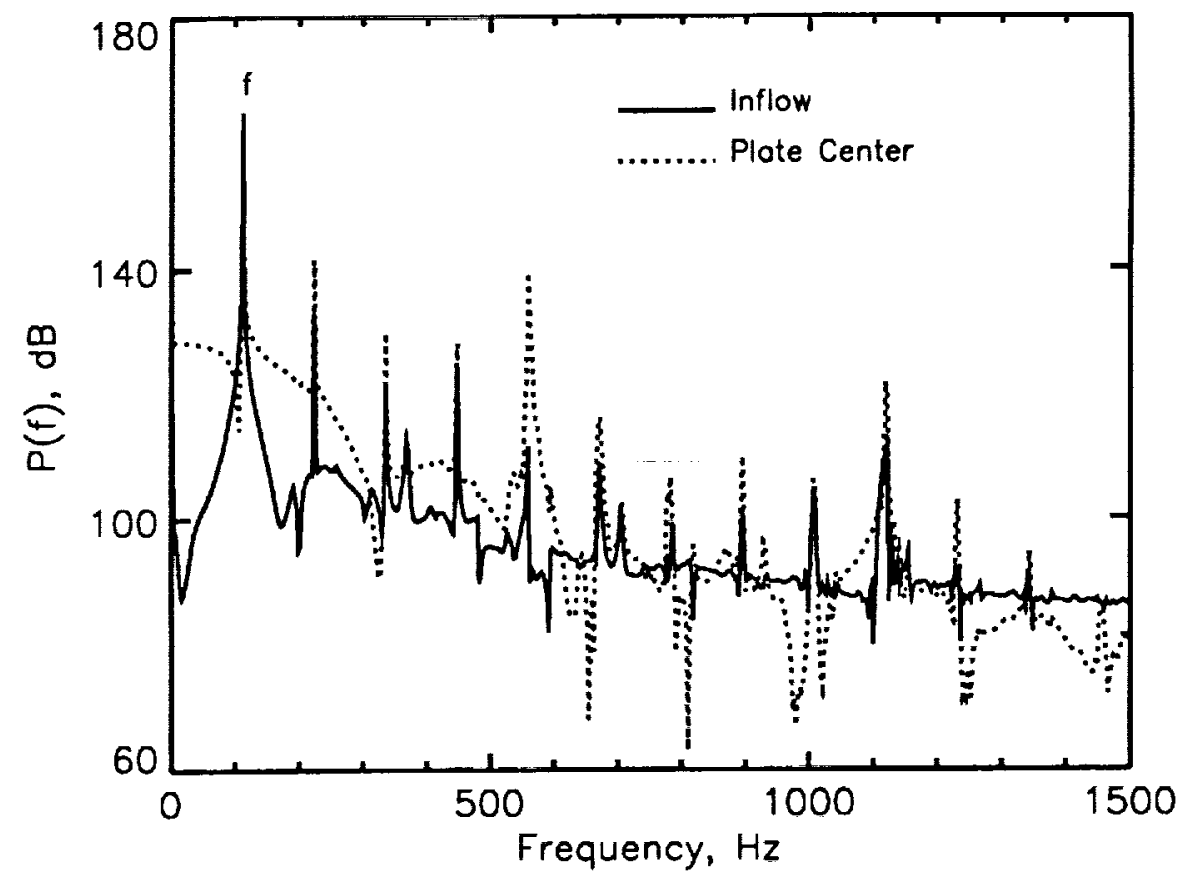

Fig. 14 Power spectra of the Top domain pressures at the inflow and near the center of the flexible plate. 


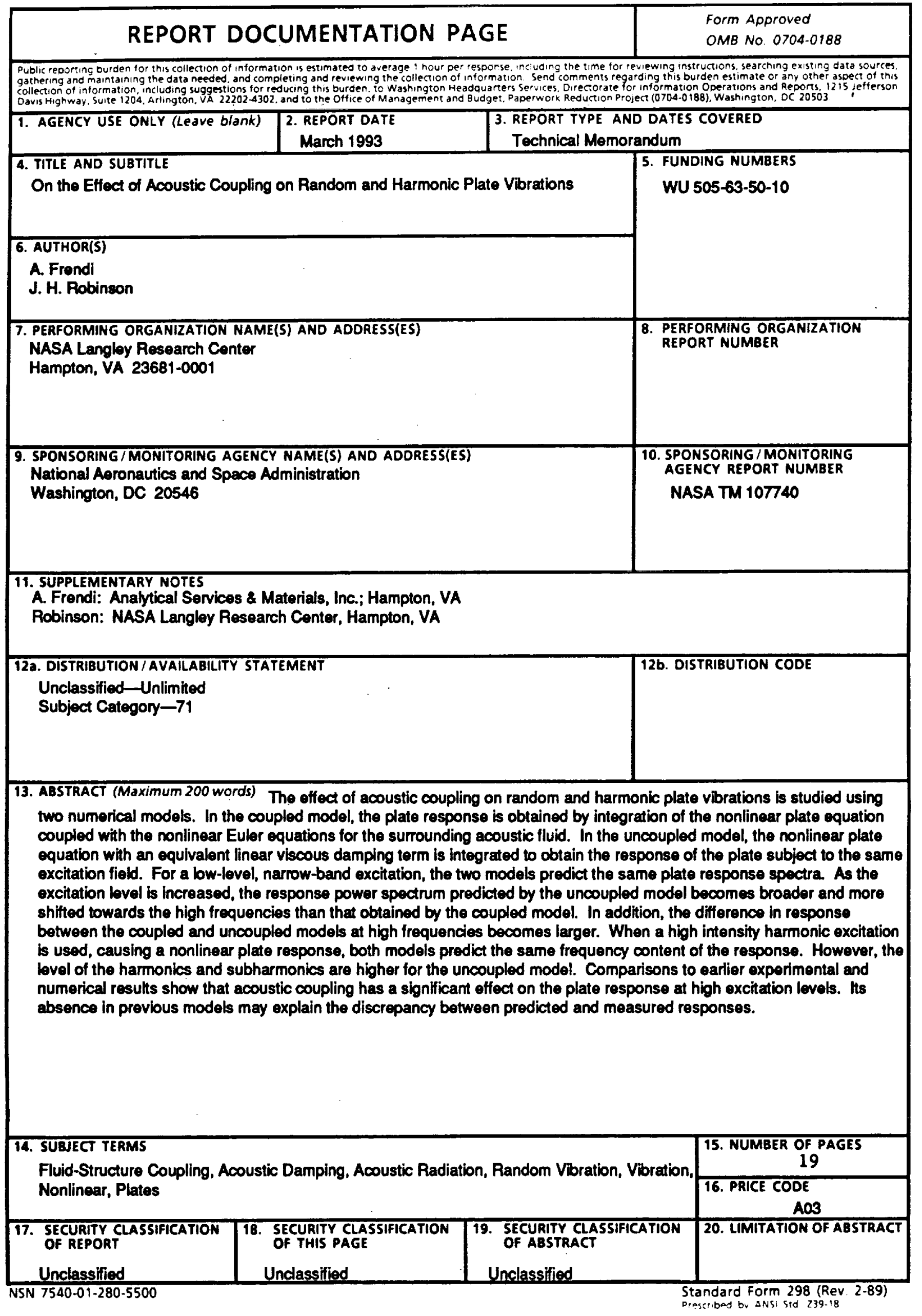


